\title{
Theory of Nuclear Reactions
}


This page is intentionally left blank 


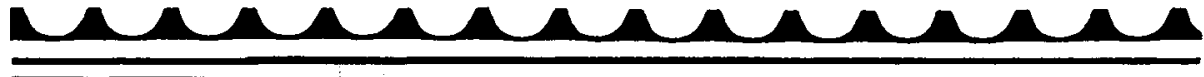 \\ Theory of Nuclear Reactions
}

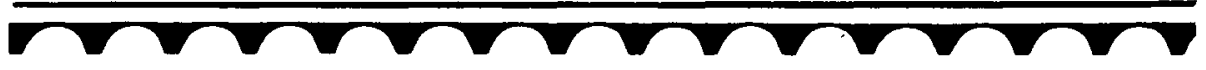

\author{
A G Sitenko \\ Institute for Theoretical Physics \\ Academy of Sciences of the Ukrainian SSR \\ Kiev, USSR \\ Translator \\ O D Kocherga
}




\section{Published by}

World Scientific Publishing Co. Pte. Ltd.

P O Box 128, Farrer Road, Singapore 9128

USA office: 687 Hartwell Street, Teaneck, NJ 07666

UK office: 73 Lynton Mead, Totteridge, London N20 8DH

\section{THEORY OF NUCLEAR REACTIONS}

Copyright $\odot 1990$ by World Scientific Publishing Co. Pte. Ltd.

All rights reserved. This book, or parts thereof, may not be reproduced in any form or by any means, electronic or mechanical, including photocopying, recording or any information storage and retrieval system now known or to be invented, without written permission from the Publisher.

ISBN $9971-50-481-2$

$9971-50-482-0$ (pbk) 
To my dear daughter Alla 
This page is intentionally left blank 


\section{PREFACE}

This book is an extended version of the second part of the lecture course on nuclear theory which has been given by the author for some years at the physical faculty of Kiev State University. The first part deals with the nuclear structure. It was published before, Ref. 1. The second part considers the nuclear reactions. It concerns the nonrelativistic theory; problems of relativistic nuclear physics are not discussed.

The nonrelativistic theory of nuclear reactions, as well as the nonrelativistic scattering theory, ${ }^{2}$ essentially employ the scattering matrix (S-matrix) concept. Since no consistent microscopic theory has been worked out up to now, the scattering matrix is obtained phenomenologically under special assumptions about the nuclear interaction nature. Nuclear processes can occur through a variety of mechanisms. Among these, compound-nucleus reactions and direct nuclear reactions are the most important. The book gives a detailed discussion of reaction mechanisms and considers their relationship. Much attention is paid to the R-matrix theory and its applications to the description of resonance nuclear processes, as well as to different approaches (the dispersion method among them) to the study of direct nuclear reactions. A detailed account is given of both the optical model, describing low-energy nuclear processes, and the diffraction phenomena which accompany nucleon-nucleus interactions. Few-body nuclear reactions, in particular, three-particle processes, are discussed. The dispersion theory treating direct nuclear reactions in terms of Feynman graphs, is considered in detail. Highenergy diffraction processes involving composite nuclear particles are studied by means of multiple scattering theory.

Though the present theory of nuclear reactions is phenomenological and has some inherent disadvantages, it provides, nevertheless, a satisfactory description of nonrelativistic nuclear processes and a reliable ground for practical calculations in applied physics and nuclear power engineering. Though present settled understanding of nuclear processes 
has been developed for several decades and discussed in a number of monographs, no consistent and sufficiently detailed account of the theory of nuclear reactions has been written up to now. This prompted the author to propose the present book. The main attention is paid to basic conceptions of the theory of nuclear reactions. Nevertheless, it also covers many problems which have been considered on ly in original papers and have not been included in existing manuals. In the concluding part of the book, a list of most important books on nuclear theory ${ }^{3-15}$ and original papers is given which does not pretend, however, to be complete. Though writing this book has cost the author much time and effort, he is aware of its disadvantages and will appreciate any critical or constructive remarks.

The author is thankful to Prof. V.F. Kharchenko for his assistance in preparing certain sections of Chaps. 1 and 2 . 


\section{CONTENTS}

\section{PREFACE}

vii

Chapter 1. GENERAL DESCRIPTION OF NUCLEAR REACTIONS

1.1. Kinematics and Conservation Laws 1

Nuclear reactions

Energy and momentum conservation

Relativistic kinematics

Problems

1.2. Collision Matrix (Central Interaction) 21

Wave function asymptotics and the collision matrix 21

Unitarity of the collision matrix $\quad 29$

Cross-sections 33

Reciprocity theorem and detailed balancing 39

1.3. Collision Matrix in the Case of Spin-Possessing

Particles (Noncentral Interaction)

Spin and orbital moments addition

Wave function asymptotics and scattering and

reaction amplitudes

Parametrization of the collision matrix

Problems

Angular distributions of nuclear reaction products

1.4. Polarization Phenomena in Nuclear Reactions 61

Polarization of nuclear reaction products 61

Density matrix $\quad 66$

Spin-tensor expansion $\quad 70$

Problems $\quad 74$

Reaction amplitudes and the final state density matrix

Scattering of a spin-1/2-particle by a spinless one 80

Scattering of polarized particles 85

Double scattering $\quad 87$

Prob lems

Helicity representation $\quad 102$

$\begin{array}{ll}\text { Problems } & 111\end{array}$

Chapter 2. R-MATRIX THEORY OF NUCLEAR REACTIONS

2.1. Conditions for the Wave Function on the Interaction Region Boundary and the Collision Matrix 116 Wave functions in the external region 116 
Relation between the S-matrix and the wave function logarithmic derivative

Wave function logarithmic derivative and the cross-sections

Problems

2.2. Munction 134

Dispersion formula for the one-channel case 134

Relation between the $A$-function and the collision matrix

One-resonance approximation

Boundary-condition-dependence of the 9 -function 142

Problems

2.3. M-Matrix 152

External wave functions for the case of $N$ open channels

Eigenfunctions of the compound system

Multichannel dispersion formula

Relation between the $\mathbb{P}$-matrix and the collision matrix $S$

Breit-Wigner formula for an isolated level 161

Problems

Kapur and Peierls basis 168

Wigner basis

2.4. $\mathscr{A}$-Function Description of Particle Interactions 170

Off-energy-shell wave function 170

Off-energy-shell two-particle amplitude 175

Problems

Chapter 3. COMPOUND-NUCLEUS REACTIONS

3.1. Compound Nucleus

Two mechanisms of nuclear reactions 186

Energy spectrum of the compound nucleus 192

Decay states of the compound nucleus 196

3.2. Resonance Nuclear Processes 201

Conditions of resonance origination 201

Resonance elastic scattering $(l=0) \quad 204$

Resonance reactions $(l=0) \quad 208$

Resonance scattering and resonance reactions $(\ell \neq 0) \quad 211$

Consideration of spins 216

$\begin{array}{lr}\text { Problems } & 219\end{array}$

3.3. Resonance Neutron-Nucleus Interaction 223

Neutron-induced nuclear reactions 223

Consideration of the nuclei thermal motion 227 
Experimental measurements of neutron resonance parameters

Statistical distribution of resonance parameters 234

Problem

3.4. Average Values and Fluctuations of the Cross-Sections 242

Averaging of cross-sections 242

Fluctuations of the cross-sections 246

3.5. Nuclear Reactions Accompanied by the Compound Nucleus Formation in Continuum

Cross-sections of compound nucleus formation with energy in the continuum range

Compound nucleus formation cross-sections and decay probabilities

Compound nucleus decay

Secondary nuclear reactions

Statistical model

Problems

\section{Chapter 4. OPTICAL MODEL AND DIFFRACTION PHENOMENA}

4.1. Low-Energy Optical Mode 1280

Nucleon absorption and complex optical potential 280 Cross-section averaging over the resonances 285 Shape elastic scattering 291 The strength function 293 Problems 299

4.2. Complex Optical Potential 305

. ile form of the optical potential 305

Optical potential parameters. Consideration of 308

Particle absorption intensity spatial distribution within the nucleus

Comparison of the optical model calculations to the experimental data

Problems

4.3. Nucleon-Nucleus Diffraction Scattering 323

Diffraction approximation 323

Neutron diffraction scattering by absorbing nuclei 328

Nuclear transparency influence on the diffraction scattering

Diffraction scattering of charged particles by nuclei

Nucleon polarization under the diffraction scattering

Particle inelastic diffraction scattering by nonspherical nuclei

Problem 
4.4. Deuteron-Nucleus Diffraction Interaction 351

Diffraction phenomena under the interaction between composite particles and nuclei

Elastic diffraction scattering of deuterons

Diffraction dissociation of deuterons

cross-section

Total deuteron-nucleus interaction cross-section

Stripping reaction

364

Deuteron absorption by nuclei

Nuclear boundary diffuseness effect on the diffraction dissociation cross-section

Problems

\section{Chapter 5. DIRECT NUCLEAR REACTIONS}

5.1. Stripping Reactions $(d, p)$ and $(d, n) \quad 385$

Deuteron-induced reactions 385

Differential cross-section of the stripping 388

Plane wave approximation 396

Deuteron and proton wave scattering 400

Stripping reaction approach in the study of nuclear structure

Polarization phenomena under the stripping

403

Deuteran formation under nucleon-nucleus collisions and other direct processes involving deuterons

Problems

5.2. Direct Inelastic Scattering and Knockout of Nucleons 428

Distorted wave approximation

Nucleon-nucleus inelastic scattering

Problems

440

Nucleon knockout (by nucleons) from nuclei 454

5.3. Three-Particle Nuclear Reactions 460

Two- and three-particle nuclear processes $\quad 460$

Peculiarities of the three-particle energy spectrum 461

Impossibility of separating the interaction region in the three-particle configuration space.

Dependence of the three-particle problem on the two-particle amplitude off-energy behaviour

Approximate models of three-particle processes

Approximate approaches to the solution of the exact three-particle problem

Problems

5.4. Dispersion Theory of Direct Nuclear Reactions 484

Amplitude analyticity and Feynman graphs 484

Cross symmetry 488

Pole approximation $\quad 490$

Triangle graph $\quad 497$ 
Initial and final state interactions

Chapter 6. MULTIPLE DIFFRACTION SCATTERING

6.1. High-Energy Diffraction Nuclear Reactions 520

Introduction $\quad 520$

High-energy approximation $\quad 521$

Scattering by a system of bound particles $\quad 525$

Compensation of multiple scattering effects $\quad 530$

Nuclear center-of-mass motion 534

Elastic scattering amplitude and optical limit 536

Inelastic scattering 541

Integral cross-sections of diffraction processes 543

Problems

545

6.2. High-Energy Nucleon-Nucleus Scattering 555

Parametrization of high-energy two-nucleon interaction

555

Nucleon-deuteron scattering 558

Nucleon-nucleus scattering 564

Multiple scattering effects and impulse approximation 571

Inelastic screening in high-energy diffraction processes

Problems

REFERENCES

601

SUBJECT INDEX 